ORIGINAL ARTICLE

\title{
Ergonomic stressors and upper extremity musculoskeletal disorders in automobile manufacturing: a one year follow up study
}

\author{
L Punnett, J Gold, J N Katz, R Gore, D H Wegman
}

Occup Environ Med 2004;61:668-674. doi: 10.1136/oem.2003.008979

See end of article for authors' affiliations

Correspondence to:

Professor L Punnett,

Department of Work Environment, University of Massachusetts Lowell, One University Avenue, Lowell, MA 01854, USA:

Laura_Punnett@uml.edu

Accepted 12 March 2004

\begin{abstract}
Aims: To estimate the one year cumulative incidence and persistence of upper extremity (UE) soft tissue disorders, in a fixed cohort of automotive manufacturing workers, and to quantify their associations with ergonomic exposures.

Methods: At baseline and at follow up, cases of UE musculoskeletal disorders were determined by interviewer administered questionnaire and standardised physical examination of the upper extremities. The interview obtained new data on psychosocial strain and updated the medical and work histories. An index of exposure to ergonomic stressors, obtained at baseline interview, was the primary independent variable. Cumulative incidence and persistence of UE disorders (defined both by symptoms and by physical examination plus symptoms) were analysed in relation to baseline ergonomic exposures, adjusting for other covariates. The incidence of new disorders was modelled using multivariate proportional hazards regression among workers who were not cases in the first year and the prevalence on both occasions was modelled by repeated measures analysis.

Results: A total of 820 workers (69\% of eligible cohort members) was examined. Follow up varied slightly by department group but not by baseline exposure level or other characteristics. Among the non-cases at baseline, the cumulative incidence of UE disorders was $14 \%$ by symptoms and $12 \%$ by symptoms plus examination findings. These rates increased with index of physical exposures primarily among subjects who had the same jobs at follow up as at baseline. Increased exposure during follow up increased risk of incidence. The persistence of UE disorders from baseline to follow up examination was nearly $60 \%$ and somewhat associated with baseline exposure score.

Conclusions: These longitudinal results confirm the previous cross sectional associations of UE musculoskeletal disorders with exposure to combined ergonomic stressors. The exposure-response relation was similar for incident cases defined by symptoms alone and those confirmed by physical examination.
\end{abstract}

$\mathrm{T}$ he epidemiological literature on musculoskeletal disorders (MSDs) in relation to occupational exposures consists primarily of cross sectional rather than prospective studies. ${ }^{1-3}$ Until recently there have been few longitudinal studies of occupational risk factors for upper extremity MSDs, and many of those addressed psychosocial stressors exclusively. The cross sectional literature documents numerous associations with physical exposures, but methodological concerns about these studies include the temporal relation of exposure to outcomes; information bias, especially when symptoms and exposures are collected simultaneously by self report (common instrument bias); over-representation of long duration cases (length biased sampling); and underestimation of effect due to potential selection bias ("healthy worker effect"). ${ }^{4}$ Further, knowledge remains sparse as to the factors that predict recovery or persistence among workers who continue in their jobs after onset of a musculoskeletal disorder. $^{5}$

In a cross sectional study of ergonomic stressors and upper extremity musculoskeletal disorders (UE MSDs) in automotive manufacturing, 1314 workers had been surveyed in selected production areas of one engine plant and one stamping plant. ${ }^{6}$ Upper extremity, shoulder, and wrist/hand disorders were all associated with exposure to physical ergonomic stressors in the subjects' usual jobs. A follow up survey was conducted about one year later. The primary objective was to examine whether the same occupational ergonomic exposures associated with UE disorders cross sectionally were also predictors of occurrence of UE disorders among previous non-cases and of MSD persistence among baseline cases, thus resolving the temporal direction of the previously reported associations.

\section{SUBJECTS AND METHODS \\ Study population}

The study was conducted at one automotive stamping plant (SP) and one automobile engine assembly plant (EP), both in Detroit, Michigan (USA). Of the 1314 workers originally surveyed, ${ }^{6} 1210$ were sought for follow up. Exclusions were for poor baseline data quality or cooperation; inability to be fully examined because of prior injury (for example, amputation); and non-production work duties (for example, full time union officers). These exclusions were made blinded to both exposure and health status.

\section{Morbidity data}

Follow up interviews and examinations were carried out in 1993-94, using a similar protocol to the baseline study. All workers were interviewed and examined in work time.

Abbreviations: $B M I$, body mass index; $E P$, engine assembly plant; GEE, generalised estimating equation; JCQ, Job Content Questionnaire; MSD, musculoskeletal disorder; $P E$, physical examination; RR, relative risk; $S P$, stamping plant; UE, upper extremity 


\section{Main messages}

- In this manufacturing workforce, about $10 \%$ of participants without symptoms or physical examination findings at baseline developed new disorders within a period of about one year.

Written informed consent was obtained at the beginning of each examination. The study protocol was approved by the Institutional Review Board of the University of Massachusetts Lowell.

A standardised, interviewer administered questionnaire obtained demographic and personal characteristics such as height, weight, prior acute injuries, surgery, relevant systemic diseases, alcohol intake, tobacco use, sports, and hobbies. Subjects reporting musculoskeletal symptoms in the year preceding the interview were asked additional questions such as year of onset, symptom frequency, and duration within the past year.

A structured physical examination consisted of active range of motion manoeuvres, to screen for discomfort and restriction of motion; abnormal findings triggered corresponding passive range of motion manoeuvres. The examination also included selected resisted motions, Phalen's test for carpal tunnel syndrome, and Finkelstein's test for de Quervain's disease. The examiner was blinded to questionnaire responses and the interviewer to examination findings. Both staff members were blinded to the baseline associations and the procedure for constructing the exposure score from questionnaire responses.

Two case definitions were applied, each of which required a report of upper extremity musculoskeletal pain or discomfort within the past year. The "symptom case" required symptoms on at least 12 occasions or for at least one week within the past year, while the "physical examination (PE) case" required symptoms during the past year plus one or more findings on examination of the same body part. Both at baseline and at follow up, a subject who met a case definition at one or more locations (neck, shoulder/upper arm, elbow/ forearm, wrist/hand) was considered to have an upper extremity disorder. Because of statistical power considerations, most analyses were conducted for cases at all UE regions combined.

\section{Occupational exposures}

Baseline questionnaire data had included ratings of the physical features of subjects' usual jobs. Eight psychophysical items covered intensity of exposure to non-neutral postures, work pace, vibration, manual forces to handle tools and parts, and mechanical pressure concentrations from hand held tools in the subject's usual job. The Borg CR-10 scale ${ }^{7}$ was used to grade subjects' responses, with instructions from the interviewer to interpret the scale anchors in terms that were relevant to the specific exposure dimension. Each measure was re-scored from the original 10 point scale to a $0-3$ scale. ${ }^{6}$ There was also a single dichotomous item on machine pacing $($ no $=0$, yes $=1)$. Correlations among the nine exposure items prevented analysis of their separate effects, ${ }^{8}$ so their sum was used as an index of upper extremity ergonomic exposures (Cronbach $\alpha=0.72$ ). The exposure index was divided into equal width quartiles $(0-6,7-12,13-18$, and 19-25).

In the follow up interview the posture intensity ratings were replaced with posture frequencies for better correspondence with observed postural frequencies. The five items that were comparable between the two surveys were summed and

\section{Policy implications}

- Reducing occupational ergonomic exposures such as repetitive work, non-neutral postures, and forceful exertions would protect against both new and persisting upper extremity musculoskeletal morbidity.

each individual's difference in score was calculated from baseline to follow up. Subjects were asked whether or not their job assignments had changed and whether there had been any changes in the content of their job. The follow up instrument also contained the Job Content Questionnaire (JCQ) core items for assessment of the psychosocial work environment. ${ }^{9}$ Standard JCQ algorithms were used to compute job demand (five variables) and job control (nine variables); these were dichotomised at the mean values for the US male working population (30.67 for job demands and 65.92 for job control). ${ }^{10}$

\section{Statistical analysis}

All statistical analyses were performed with the SAS software package for personal computer. ${ }^{11}$ One-year cumulative incidence, for each case type, was computed among the baseline non-cases. Persistence of UE MSD from baseline to follow up was defined as the probability that a worker with any upper extremity disorder at baseline also had a disorder of any region of the upper extremity at follow up.

Since time to onset was not obtained on a prospective basis, it was treated as uniform across all subjects with new UE MSDs during the follow up period. The proportional hazard regression procedure, modified as per Breslow, ${ }^{12}$ was used for multivariate analysis of UE MSD cumulative incidence and persistence. A four level categorical variable was used to represent the physical exposure index, using the first quartile (0-6, "very low") as the reference value for the "low", 7-12 "moderate", ${ }^{13-18}$ and "high"19-25 quartiles. Covariates included: gender; age (three strata); plant (EP/ SP); high seniority (25+ years); high Quetelet body mass index $\left(\mathrm{kg} / \mathrm{m}^{2}\right)(B M I \geqslant 26)$; previous acute injury to the upper limb; systemic disease; and weekly participation in second jobs, sports, or other recreational activities (all yes/no). Retention of covariates was based primarily on whether or not they changed other point estimates by at least $15 \%$, although coefficients with p values above 0.15 generally were excluded.

An alternative statistical approach was employed to avoid the assumptions implicit in the terms of "cumulative incidence" (first onset of an irreversible condition) and "persistence" (continuous presence of pain from one survey occasion to the next). Multivariate generalised estimating equation (GEE) regression for repeated measures (SAS Proc Genmod) was employed to model the prevalence of PE disorders in each survey as a function of exposure level at the same occasion, with unstructured correlations and log link to estimate relative risk. Repeated measures analysis takes into account the within subject correlation between case statuses at the two survey occasions; ${ }^{13}$ that is, case status at follow up was conditional on baseline case status.

\section{RESULTS}

Thirty hourly workers sought for follow up were deceased, retired, transferred out of the study plants, or changed from hourly to salaried status during the follow up interval. Of the remaining 1180 eligible for follow up, 820 (69\%) were examined. The follow up interview and examination took 
Table 1 Follow up survey of automotive stamping plant and engine plant workers, Detroit, MI, USA, 1993-94; population examined at follow up, as a proportion of eligible baseline subjects, by baseline exposure level*

\begin{tabular}{lcc}
\hline Exposure level & Baseline $(\mathbf{n})$ & $\begin{array}{l}\text { No. }(\%) \text { examined at } \\
\text { follow up }\end{array}$ \\
\hline Missing & 127 & $76(60 \%)$ \\
Very low (0-6) & 116 & $76(66 \%)$ \\
Low (7-12) & 344 & $251(73 \%)$ \\
Moderate (13-18) & 468 & $336(72 \%)$ \\
High (19-25) & 125 & $61(65 \%)$ \\
Total & 1180 & $820(69 \%)$ \\
\hline
\end{tabular}

${ }^{*} \mathrm{p}$ value $=0.19$ from $\chi^{2}$ statistic on 3 d.o.f., test of difference in proportions among non-missing exposure levels.

place $44 \pm 6$ (median 45, range 26-66) weeks after the baseline survey.

Follow up participation was the same between the two plants and by baseline case status, seniority, and gender. There was no trend in participation by baseline exposure (table 1).

Of the 820 subjects examined at follow up, 30 were excluded because of language or comprehension difficulties or unreliable responses. The 790 remaining participants had similar demographic and work history characteristics as the study population at baseline (table 2). The workforce was largely male and middle aged, and more than half was African-American.

Most follow up subjects (744 of 790) had provided physical exposure data at baseline. For the sum of physical exposure items asked identically on both surveys, individual between survey differences ranged from -10 to 10 but averaged about zero. More than one fourth of workers reported a change in either job assignment or job content during the follow up period. Those who reported changed job assignments had slightly decreased exposure scores (mean $-0.7 \pm 3.2$ ), in contrast to those reporting no job change (mean $0.1 \pm 2.6$ ) $(p=0.03)$. Two thirds of participants providing exposure data on both surveys remained in the same exposure quartile, while $17 \%$ moved to a higher quartile and $16 \%$ to a lower one. Only eight subjects, all cases at baseline, reported job changes related to their MSD status.

The baseline physical ergonomic index was moderately correlated with psychological job demands $(r=0.32$, $\mathrm{p}<0.0001)$ at follow up but not at all with job control $(p=0.51)$. Among individual items, the psychophysical rating of work pace was associated with the JCQ items, "work very hard" $(r=0.26)$ and "work very fast" $(r=0.25)$. About one fourth of the subjects were in jobs with "high" psychological demands and about one half in "low control" jobs, but only $15 \%$ (110) were in jobs with both high demands and low control. Workers with baseline physical exposures above the middle of the scale (13-25) were 2.4 times more likely to be in high strain versus low strain jobs (95\% CI 1.6 to 3.7 ).

\section{Cumulative incidence}

In the follow up population, 247 (31\%) had an upper extremity disorder by symptom history and 215 (28\%) had an upper extremity PE disorder. As in the baseline survey, prevalences for the wrist/hand and shoulder/upper arm were about double those of the neck or elbow.

The subjects who were not symptom or PE cases at baseline were considered at risk for the corresponding "incident" disorders at follow up. Of each at-risk group, about $90 \%$ had provided baseline exposure data and about $75 \%$ reported no change in job assignment or job content at follow up.

The cumulative incidence of new disorders was $14 \%$ for symptom cases $(n=68)$ and $12 \%$ for PE cases $(n=62)$. Incidence by UE region ranged from 3\% (neck) to 7\% (wrist/ hand).

By either case definition, frequency of new UE disorders was nearly doubled in workers with physical job exposure score of 13-25, those with high demand/low control jobs by JCQ, women, and people with high BMI. Marginal increases were observed in white workers, those who were younger

Table 2 Baseline demographic characteristics and medical histories of automotive stamping plant and engine plant workers; total baseline cohort and subjects with reliable data in follow up survey, Detroit, MI, USA, 1993-94

\begin{tabular}{|c|c|c|c|c|}
\hline & \multirow{2}{*}{$\begin{array}{l}\text { Baseline } \\
\text { Total } \\
(n=1283)\end{array}$} & \multicolumn{3}{|l|}{ Follow up } \\
\hline & & $\begin{array}{l}\text { Total } \\
(n=790)\end{array}$ & $\begin{array}{l}\text { Non-case at } \\
\text { baseline } \\
(\mathrm{n}=458)\end{array}$ & $\begin{array}{l}\text { Case at } \\
\text { baseline } \\
(n=332)\end{array}$ \\
\hline Stamping plant: no. (\%) & $670(52 \%)$ & $414(53 \%)$ & $221(48 \%)$ & $193(58 \%)$ \\
\hline Male: no. (\%) & $1047(82 \%)$ & $643(81 \%)$ & $397(87 \%)$ & $246(74 \%)$ \\
\hline Previous UE injury: no. (\%) & $509(40 \%)$ & $319(40 \%)$ & $170(37 \%)$ & $149(45 \%)$ \\
\hline Systemic disease*: no. (\%) & $266(21 \%)$ & $164(21 \%)$ & $79(17 \%)$ & $85(26 \%)$ \\
\hline Regular weekly outside activity†: no. (\%) & $846(66 \%)$ & $533(67 \%)$ & $295(64 \%)$ & $238(72 \%)$ \\
\hline \multicolumn{5}{|l|}{ Cigarette smoking: } \\
\hline no. $(\%)$ current & $577(45 \%)$ & $353(45 \%)$ & $194(42 \%)$ & $159(48 \%)$ \\
\hline no. (\%) former & $298(23 \%)$ & $195(25 \%)$ & $109(24 \%)$ & $86(26 \%)$ \\
\hline \multicolumn{5}{|l|}{ Alcohol use: } \\
\hline no. (\%) current & 753 (59\%) & $477(61 \%)$ & $259(57 \%)$ & $218(66 \%)$ \\
\hline no. (\%) former & $189(15 \%)$ & $102(13 \%)$ & $67(15 \%)$ & $35(11 \%)$ \\
\hline \multicolumn{5}{|l|}{ Racet: } \\
\hline no. (\%) African-American & - & $463(59 \%)$ & $273(60 \%)$ & $190(58 \%)$ \\
\hline no. (\%) white/non-Hispanic & - & $290(37 \%)$ & $158(35 \%)$ & $132(40 \%)$ \\
\hline Age (years): mean (SD) & $46.5(8.2)$ & $46.3(8.2)$ & $46.2(8.6)$ & $46.6(7.6)$ \\
\hline Quetelet body mass index: mean (SD) & $27.5(4.9)$ & $27.3(4.5)$ & $27.1(4.5)$ & $27.6(4.6)$ \\
\hline Seniority in company (years): mean (SD) & $21.5(6.5)$ & $21.5(6.8)$ & $21.4(7.4)$ & $21.6(5.8)$ \\
\hline Seniority in plant (years): mean (SD) & $16.9(8.1)$ & $16.8(8.2)$ & $16.6(8.5)$ & $17.0(7.6)$ \\
\hline Seniority in job (years): mean (SD) & $13.1(7.8)$ & $13.1(7.8)$ & $12.8(7.9)$ & $13.5(7.5)$ \\
\hline Ergonomic exposure score: mean (SD) & $13.1(4.8)$ & $13.1(4.7)$ & $12.3(4.9)$ & $14.1(4.2)$ \\
\hline Psychosocial job demandsł: mean (SD) & - & $28.9(3.2)$ & $28.8(3.1)$ & $29.1(3.4)$ \\
\hline Psychosocial job controlł: mean (SD) & - & $61.1(9.3)$ & $61.7(9.3)$ & $60.3(9.2)$ \\
\hline
\end{tabular}


than 40, and those with history of acute upper extremity injury or systemic disease. Incidence of new PE disorders only was higher in workers with job changes $(R R=1.7)$ and current smokers ( $R R=1.7$ versus former or never smokers). There was no association with alcohol consumption or nonwork activities.

The frequency of new UE disorders increased with exposure quartile (Mantel test of trend, $p=0.03$ for symptom cases and $\mathrm{p}=0.06$ for $\mathrm{PE}$ cases). The association with baseline exposure level was weak among participants with changed job assignment or content between baseline and follow up. However, among workers who stayed in the same jobs, there was a marked exposure-response trend in crude rates of new disorders (tests of linear trend: symptom cases, $\mathrm{p}=0.001$; PE cases, $\mathrm{p}=0.004$ ) (fig 1). The cumulative incidence of new upper extremity disorders, by each case definition, was more than four times higher in the highest compared with the lowest quartile of baseline ergonomic exposure. The risk ratio for $\mathrm{PE}$ cases from a simple Cox regression was $1.5(1.05-2.20)$ per exposure quartile in this subgroup.

In multivariable regression models, the risk of new UE disorders increased by about $50 \%$ with each quartile of the physical exposure index (table 3, model 1). Similar results were obtained for the symptom-only cases. As already observed, the overall risk was higher among those with job changes at follow up but the exposure index had less predictive value for new cases among these subjects. The
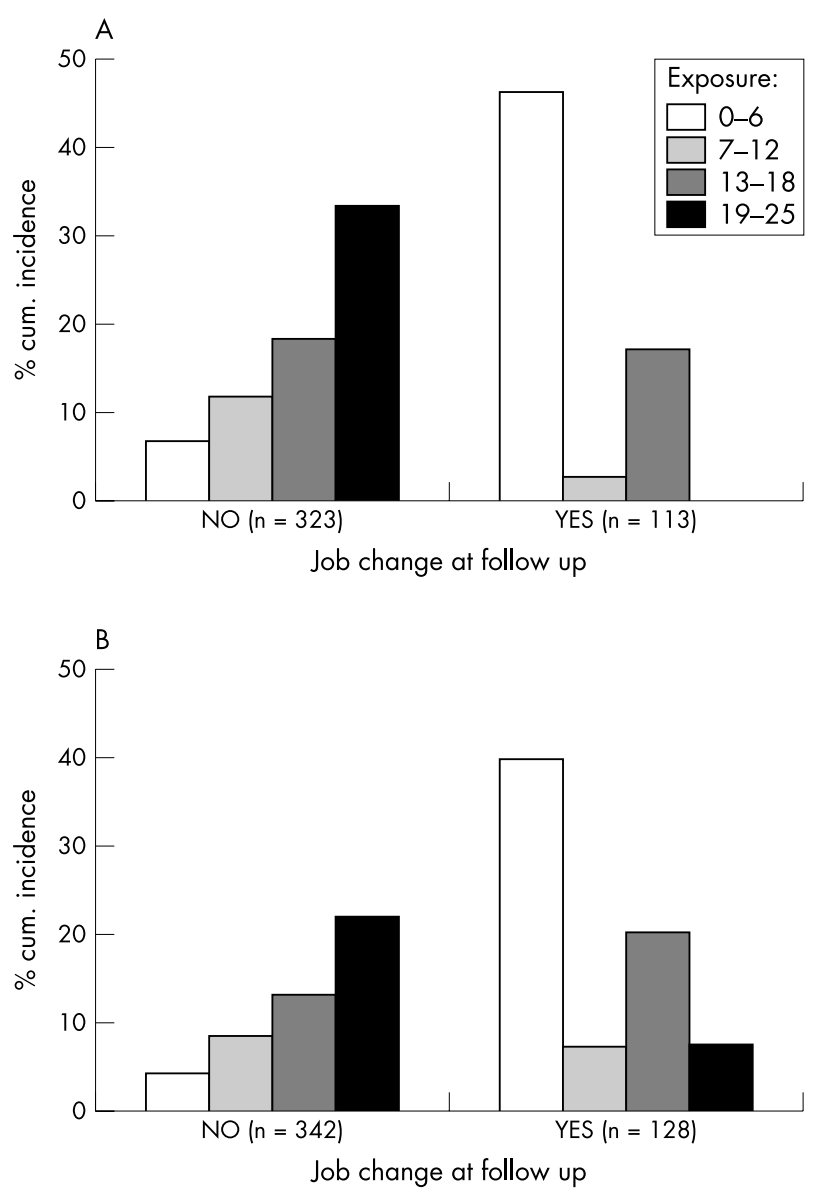

Figure 1 Cumulative incidence of new upper extremity disorders, by quartile of baseline ergonomic exposure score and job change from baseline to follow up survey; automotive stamping and engine plants, 1993-94. (A) Symptom cases; (B) physical examination cases (symptoms plus examination findings). relative risk was estimated at 3.2 for the highest exposure quartile compared with the lowest. Inclusion of high body mass index and high demand/low control did not affect the coefficients for other covariates. In separate models, the risk increased $(\mathrm{RR}=1.5)$ for workers who changed to a higher exposure quartile during follow up and decreased $(R R=0.8)$ for those who moved to a lower quartile, in addition to the effect of baseline exposure.

Restricting the analysis to subjects with no job changes (model 2) did not materially change the relative risks. In this subgroup, the most parsimonious model for symptom cases included only exposure quartile ( $R R=1.6,95 \%$ CI 1.1 to 2.2 ) and baseline PE findings ( $\mathrm{RR}=2.7,95 \% \mathrm{CI} 1.4$ to 5.1$)$. Restricting to those in the same exposure quartile at both surveys $(n=302)$ further reduced statistical power and explained less variability (likelihood ratio test $\mathrm{p}=0.38$ ), suggesting that the effect of job changes could not be explained solely by changes in exposure score.

\section{Persistence}

A total of 301 workers were symptom cases at baseline and 265 were PE cases. Again, about $90 \%$ had provided baseline exposure data and about $70 \%$ had the same job assignment and job content at follow up. Among cases who could recall date of onset, $71 \%$ had been symptomatic for more than one year at baseline (mean 1951, median 1090 days).

The overall persistence of upper extremity disorders was $59 \%$ for both symptom cases $(n=179)$ and PE cases $(n=147)$. Persistence was higher for shoulder and wrist/ hand disorders (about 40-50\%) than the neck or elbow (25$35 \%)$. By either case definition, it was slightly higher in workers who were 50 years old or more versus 20-39 and also in those whose jobs had not changed at follow up (all $\mathrm{p}$ values $>0.2$ ). Symptom cases were less likely to persist among African-American (56\%) than white $(68 \%)$ workers $(p=0.04)$ and among former smokers than either current or never smokers $(p=0.05)$. In contrast, never drinkers had the lowest persistence and former drinkers had the highest. All associations were less pronounced for PE cases.

Persistence was markedly lower in the lowest quartile of baseline exposure (tests of linear trend: symptom cases, $p=0.08$; PE cases $p=0.13$ ). These associations did not vary much with stratification on job change between surveys, except that the drop in the highest quartile was more pronounced in those with job changes (fig 2). No meaningful multivariate models could be fit to the persistence data for either UE case type.

\section{Repeated measures analysis}

The magnitude of the association with ergonomic exposure index was similar for baseline prevalence, ${ }^{6}$ cumulative incidence of new UE disorders, and the repeated measures analysis, when the same covariates were included in the model (table 4). The cumulative incidence model had a slightly larger coefficient for gender and smaller effect of systemic disease than the other two models.

\section{DISCUSSION}

In this fixed cohort of automotive manufacturing workers, the prevalence of upper extremity musculoskeletal disorders was similar at follow up to that found in the baseline survey of the same population. MSD cases were defined both on the basis of symptoms uniquely and also by the combination of symptoms plus physical examination findings. Although disorders by symptoms alone were slightly more frequent than disorders confirmed by physical examination, the two case types had quite similar risk factors. The cumulative incidence of new disorders was strongly associated with previously assessed level of exposure to combined ergonomic 
Table 3 Cumulative incidence of upper extremity disorders on physical examination; risk ratios $(95 \% \mathrm{Cl})$ from multivariate Cox regression models; automotive stamping and engine plant workers, Detroit, MI, 1993-94

\begin{tabular}{|c|c|c|}
\hline & Model 1 & Model 2 \\
\hline & $\begin{array}{l}\text { All workers at risk } \\
(\mathrm{n}=459)\end{array}$ & $\begin{array}{l}\text { Workers with no job } \\
\text { change during follow up } \\
\text { period } \\
(\mathrm{n}=335)\end{array}$ \\
\hline Exposure index quartile (1-4) & 1.5 (1.0 to 2.2$)$ & 1.5 (1.1 to 2.2$)$ \\
\hline Gender (female/male) & $1.4(0.7$ to 2.7$)$ & $1.1(0.4$ to 3.0$)$ \\
\hline Upper extremity injury (yes/no) & $1.4(0.8$ to 2.4$)$ & $1.8(0.9$ to 3.5$)$ \\
\hline Baseline physical examination findings (yes/no) & $1.7(1.0$ to 3.0$)$ & $1.6(0.8$ to 3.2$)$ \\
\hline High demand/low control (yes/no) & $1.5(0.8$ to 2.8$)$ & $1.7(0.8$ to 3.6$)$ \\
\hline High body mass index $(\geqslant 26 v<26)$ & 1.5 (0.9 to 2.7$)$ & 1.6 (0.8 to 3.2$)$ \\
\hline Job change at follow up (yes/no) & $4.8(1.5$ to 15.4$)$ & - \\
\hline Exposure score $\times$ job change & $0.5(0.2$ to 0.9$)$ & _ \\
\hline Model $p$ value from likelihood ratio test & 0.01 & 0.02 \\
\hline
\end{tabular}

stressors affecting the upper extremity. Nearly $60 \%$ of subjects who had disorders at baseline were still (or again) cases in the second survey. We did not identify consistent predictors of persistence.

The incidence results generally confirmed our previous cross sectional findings. "Length biased sampling" means that a cross sectional study necessarily identifies cases of longer duration than average, which could be either more
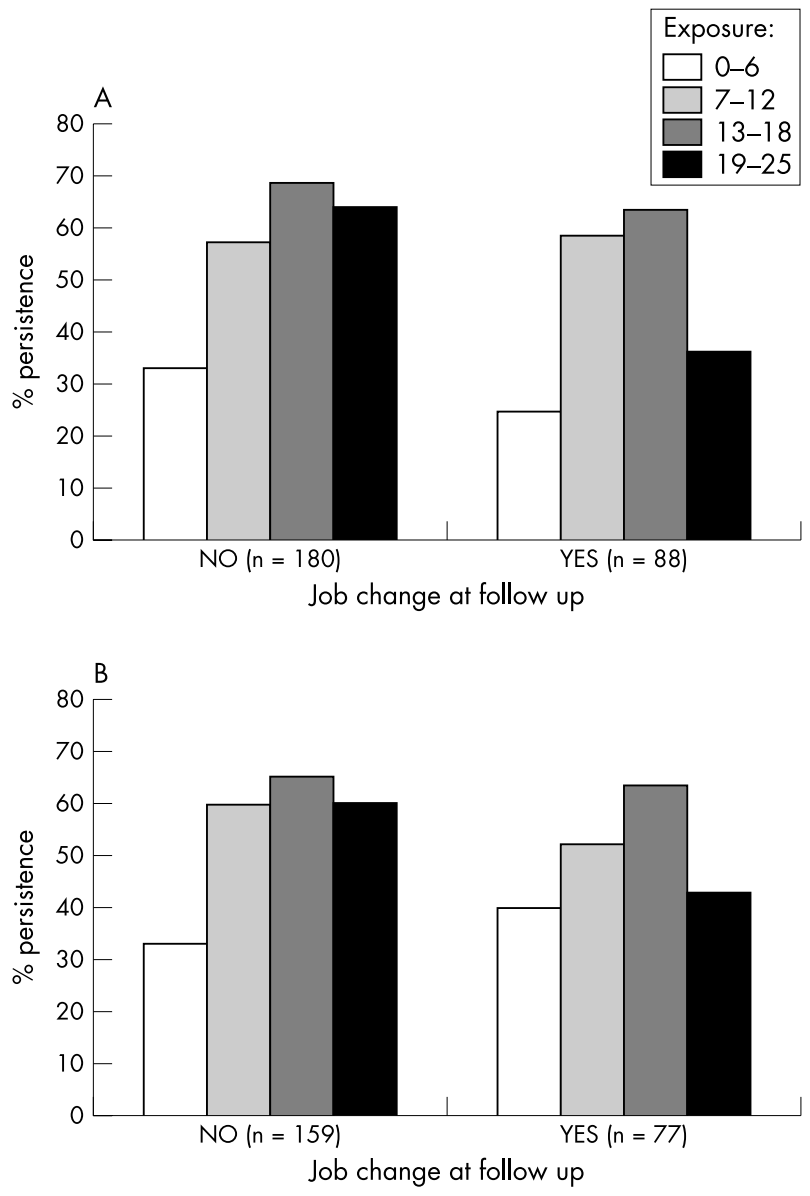

Figure 2 Persistence of upper extremity disorders, by quartile of baseline ergonomic exposure score and job change from baseline to follow up survey; automotive stamping and engine plants, 1993-94. (A) Symptom cases; (B) physical examination case (symptoms plus examination findings). sensitive (if lower thresholds) or less sensitive (if long latency period) to recent exposures than cases of new onset. In this population, there was no evidence of such bias operating in either direction in the baseline study. The relative risk for cumulative incidence was similar to what had been estimated from the prevalence data, ${ }^{6}$ with a relative risk of 1.5 per quartile of the exposure index and $\mathrm{RR}=3$ for the highest quartile compared with the lowest.

The magnitude of the relative risk estimates is striking in light of the fact that that all study jobs were drawn from a single, highly engineered industry. Even at baseline these subjects were already middle aged and had been employed for two decades, on average, in automobile manufacturing. Thus the population variance in exposure was necessarily reduced relative to the range of ergonomic exposures expected across all sectors of the economy.

Change in job assignment or job content appeared to confer an increased risk for development of new disorders, even when the exposure score was unchanged. This finding deserves further investigation. Only a small proportion of reported job changes were apparently accommodation of upper extremity symptoms; most were attributed to modernisation of production technology and consequent changes in work organisation. Subjects with ergonomic exposure ratings on both surveys changed very little over the follow up interval, and those that did were slightly lower on average in the new jobs, particularly among workers who had higher exposures at baseline. Persistence was lower among baseline cases whose jobs had changed-a finding more consistent with a potential benefit of the lower exposures, however small the reductions.

We find no obvious explanation for the apparent discrepancy that job change was a risk factor for incidence but a protective factor for persistence. It is possible that the risk of new disorders is influenced by psychosocial aspects of how production technology changes are implemented, although JCQ scores were the same for workers with and without job change, and psychosocial strain was included in the multivariable regression models. Alternatively, if job change was injurious, persistence may have been affected by the factors determining who did or did not remain in the most physically strenuous jobs. Also suggestive of a "healthy worker effect" was the slight decrease in persistence of UE disorders from baseline to follow up in the highest exposure stratum, in the presence of an otherwise strong exposure-response relation.

The combination of high demands with low control at follow up was associated with incidence but not persistence of UE MSDs. However, psychosocial strain was not assessed at baseline, so its equivocal associations with UE MSDs 
Table 4 Relative risks (95\% Cl) for baseline prevalence, prevalence (repeated measures) on two surveys, and one-year cumulative incidence of UE MSD cases on physical examination, among non-cases at baseline; workers with exposure ratings on both surveys, two automobile manufacturing plants, Detroit, MI

\begin{tabular}{|c|c|c|c|}
\hline & \multicolumn{2}{|l|}{ Prevalence } & \multirow{2}{*}{$\begin{array}{l}\text { Cumulative incidence } \\
\text { Follow up survey } \\
(n=470)\end{array}$} \\
\hline & $\begin{array}{l}\text { Baseline only } \\
(n=714)\end{array}$ & $\begin{array}{l}\text { Both surveys: } \\
\text { repeated measures } \\
(n=1855)^{*}\end{array}$ & \\
\hline $\begin{array}{l}\text { Ergonomic exposure index } \\
\text { (quartiles) }\end{array}$ & $1.3(1.1$ to 1.5$)$ & $1.3(1.2$ to 1.4$)$ & $1.2(0.9$ to 1.6$)$ \\
\hline UE injury (yes/no) & $1.2(0.9$ to 1.6$)$ & 1.1 (1.0 to 1.3 ) & 1.4 (0.8 to 2.3 ) \\
\hline Gender (male/female) & $1.7(1.2$ to 2.2$)$ & $1.6(1.4$ to 1.9$)$ & $2.0(1.1$ to 3.6$)$ \\
\hline Systemic disease (yes/no) & $1.4(1.1$ to 1.9$)$ & $1.4(1.2$ to 1.6$)$ & $1.0(0.5$ to 1.9$)$ \\
\hline
\end{tabular}

should be treated with caution. Further, unfortunately, the psychosocial aspects of job change during follow up could not be assessed. The substantial collinearity between physical and psychosocial job demands suggests that both dimensions are of concern; an integrated approach to prevention is justified in light of the role of work organisation features as the likely common upstream determinants. ${ }^{14}$

The physical examination manoeuvres used, to the extent that they have been evaluated, are considered lacking in specificity and sensitivity. ${ }^{15-17}$ The goal in utilising this examination protocol was not to assign a specific clinical diagnosis, but rather to require an examiner's finding that corresponded with the symptoms. Any errors introduced would most likely have been non-differential with respect to exposure status, since the examiner was blinded to questionnaire responses. Some specificity may also have been lost by the aggregation of all UE disorders into a combined endpoint. Of note, subjects with baseline physical examination findings but no important symptoms in the same UE region had increased risk of being cases at follow up, suggesting that some manoeuvres might have more accuracy or predictive value than others have shown.

Misclassification of ergonomic exposures, either differential or non-differential, could result from inaccurate assessment by worker self report. ${ }^{18-20}$ However, in this study, the primary index of physical exposures was obtained prospectively, making it unlikely that systematic information bias could be an alternative explanation of case status about one year later.

Among potential confounding variables, UE MSDs were not associated with sports and other recreational activities or second jobs. Covariates that were associated with at least some endpoints included gender, prior acute injury to the upper extremity, systemic disease affecting the soft tissues, and high age and/or plant seniority. However, these risk factors exerted little confounding influence, in that the RRs estimated in multivariate analyses were not notably different from the crude estimates.

The calculation of cumulative incidence implicitly requires the assumption of first onset of an irreversible condition, and "persistence" similarly implies the continuous presence of pain from one survey occasion to the next. Nevertheless, MSDs are frequently reversible or episodic in nature. Thus, an alternative statistical approach is to analyse prevalent cases, conditional on previous case status and exposures. This approach also maximises statistical power, as all observations are included rather than only the subset of subjects who were or were not cases in the previous survey. Comparison of this approach to the traditional analysis, in this population, showed very similar exposure-response relations (table 4). Hoogendoorn and colleagues ${ }^{21}$ similarly compared conven- tional logistic regression to GEE models with time dependent covariates, in a three year longitudinal study of low back pain, and reported about $15 \%$ higher odds ratios for physical occupational exposure exposures from the GEE models.

This study adds to the growing literature of prospective studies of upper extremity disorders showing that exposure to ergonomic stressors such as repetitive movements, nonneutral postures, or forceful exertions contribute to the occurrence of upper extremity MSDs in manufacturing, ${ }^{22-25}$ food processing, ${ }^{26-28}$ clerical work, ${ }^{29-32}$ health care, ${ }^{33}$ forestry, ${ }^{34}$ and mixed occupations. ${ }^{35-38}$ This evidence, further strengthened by the findings reported here, suggests that positive associations reported in cross sectional studies should not be dismissed as artefacts of that study design and that, in light of the basic science evidence, ${ }^{3}$ are consistent with a causal relation.

\section{ACKNOWLEDGEMENTS}

This study was funded by a contract from the United Auto WorkersChrysler (now Daimler-Chrysler) National Joint Committee (NJC) on Health and Safety. Its performance relied heavily on the assistance and cooperation of National Training Center staff, plant managers, safety directors, UAW ergonomics trainers, and health and safety committee members. Ms Twila Eshelman and Ms Trudy Johnson Hall conducted the physical examinations and interviews. Ms Lei Peng performed extensive data management and analysis. Dr Robert Karasek offered valuable insights regarding interpretation of the JCQ findings. Dr Rebecca Moreland has been an unflagging supporter and liaison for this project to the NJC. Dr Katz was supported by NIH \# P60 AR36308, P60 AR 47782, K24 AR 02132, and the Arthritis Foundation.

\section{Authors' affiliations}

L Punnett, J Gold, R Gore, D H Wegman, Department of Work Environment, University of Massachusetts Lowell, One University Avenue, Lowell, MA 01854, USA

J N Katz, Robert Brigham Arthritis and Musculoskeletal Clinical Research Center, Section of Clinical Sciences, Division of Rheumatology, Immunology and Allergy, Brigham and Women's Hospital, Harvard Medical School, Boston, MA, USA

\section{REFERENCES}

1 Bernard BP, ed. Musculoskeletal disorders and workplace factors: a critical review of epidemiologic evidence for work-related musculoskeletal disorders of the neck, upper extremity, and low back. Cincinnati, OH: Department of Health and Human Services, National Institute for Occupational Safety and Health, 1997.

2 Malchaire J, Cock N, Vergracht S. Review of the factors associated with musculoskeletal problems in epidemiological studies. Int Arch Occup Environ Health $2001 ; 74(2): 79-90$.

3 National Research Council, the Institute of Medicine. Musculoskeletal disorders and the workplace: low back and upper extremities. Washington, DC: National Academy Press, 2001.

4 Checkoway H, Pearce NE, Kriebel D. Research methods in occupational epidemiology. New York: Oxford University Press, 2004. 
5 Cole DC, Hudak PL. Prognosis of nonspecific work-related musculoskeletal disorders of the neck and upper extremity. Am J Ind Med 1996;29:657-68 6 Punnett L. Ergonomic stressors and upper extremity disorders in vehicle manufacturing: cross-sectional exposure- response trends. Occup Environ Med 1998;55:414-20.

7 Borg G. Psychophysical scaling with applications in physical work and the perception of exertion. Scand J Work Environ Health 1990; 16(suppl 1):55-8.

8 Punnett L, van der Beek AJ. A comparison of approaches to modeling the relationship between ergonomic exposures and upper extremity disorders. Am J Ind Med 2000;37:645-55.

9 Karasek RA. Job Content Questionnaire and User's Guide, 1985.

10 Karasek RA, Brisson C, Kawakami N, et al. The job content questionnaire (JCQ): an instrument for internationally comparative assessments of psychosocial job characteristics. J Occup Health Psychol 1998:3:322-55.

11 SAS Institute I. SAS Procedures Guide, version 6, 3rd edn. Cary NC: SAS Institute, Inc., 1990

12 Breslow N. Covariance analysis of censored survival data. Biometrics 1974:30:89-99.

13 Diggle PJ, Heagerty PJ, Liang K-Y, et al. Analysis of longitudinal data, 2nd edn. Oxford: Oxford University Press, 2002.

14 MacDonald LA, Karasek RA, Punnett L, et al. Covariation between workplace physical and psychosocial stressors: evidence and implications for occupational health research and prevention. Ergonomics 2001:44:696-718.

15 Katz JN, Larson MG, Fossel AH, et al. Validation of a surveillance case definition of carpal tunnel syndrome. Am J Public Health 1991;81:189-93.

16 Marx RG, Bombardier C, Wright JG. What do we know about the reliability and validity of physical examination tests used to examine the upper extremity? J Hand Surg (Am) 1999;24A:185-93.

17 Viikari-Juntura E, Riihimäki $\mathrm{H}$. New avenues in research on musculoskeletal disorders. Scand J Work Environ Health 1999;25(6, special issue):564-8

18 Torgén M, Winkel J, Alfredsson L, Kilbom A, Stockholm MUSIC 1 Study Group t. Evaluation of questionnaire-based information on previous physical loads. Scand J Work Environ Health 1999;25:246-54.

19 Viikari-Juntura E, Rauas S, Martikainen R, et al. Validity of self-reported physical work load in epidemiologic studies on musculoskeletal disorders. Scand J Work Environ Health 1996;22:251-9.

20 Wiktorin C, Karlqvist LK, Winkel J, Stockholm MUSIC I study group t. Validity of self-reported exposures to work postures and manual material handling. Scand J Work Environ Health 1993;19:208-14.

21 Hoogendorn WE, Bongers PM, Vet HCWd, et al. Comparison of two different approaches for the analysis of data from a prospective cohort study: an application to work related risk factors for low back pain. Occup Environ Med 2002;59:459-65.

22 Häkkänen M, Viikari-Juntura E, Martikainen R. Incidence of musculoskeletal disorders among newly employed manufacturing workers. Scand J Work Environ Health 2001;27:381-7.
23 Jonsson BG, Persson J, Kilbom A. Disorders of the cervicobrachial region among female workers in the electronics industry: a two-year follow up. Int $J$ Ind Ergon 1988;3:1-12.

24 Schibye B, Skov T, Ekner D, et al. Musculoskeletal symptoms among sewing machine operators. Scand J Work Environ Health sewing machine oper

25 Veiersted KB, Westgaard RH. Subjectively assessed occupational and individual parameters as risk factors for trapezius myalgia. Int $\mathrm{J}$ Ind Ergon 1994; 13:235-45

26 Frost $\mathbf{P}$, Andersen JH, Nielsen VK. Occurrence of carpal tunnel syndrome among slaughterhouse workers. Scand J Work Environ Health 1998:24:285-92.

27 Frost $\mathrm{P}$, Andersen JH. Shoulder impingement syndrome in relation to shoulder intensive work. Occup Environ Med 1999;56:494-8.

28 Olafsdóttir H, Rafnsson V. Increase in musculoskeletal symptoms of upper limbs among women after introduction of the flow-line in fish-fillet plants. Int J Ind Ergon 1998;21:69-77.

29 Bergqvist U. Visual display terminal work-a perspective on long-term changes and discomforts. Int J Ind Ergon 1995;16:201-9.

30 Ferreira JM, Conceicao GM, Saldiva PH. Work organization is significantly associated with upper extremities musculoskeletal disorders among employees engaged in interactive computer-telephone tasks of an international bank subsidiary in Sao Paulo, Brazil. Am J Ind Med 1997;31:468-73

31 Korhonen T, Ketola R, Toivonen R, et al. Work related and individual predictors for incident neck pain among office employees working with video display units. Occup Environ Med 2003;60:475-82.

32 Marcus M, Gerr F, Monteilh C, et al. A prospective study of computer users: II. Postural risk factors for musculoskeletal symptoms and disorders. Am J Ind Med 2002;41:236-49.

33 Smedley J, Inskip H, Trevelyan F, et al. Risk factors for incident neck and shoulder pain in hospital nurses. Occup Environ Med 2003;60:864-9.

34 Miranda $\mathrm{H}$, Viikari-Juntura E, Martikainen $\mathrm{R}$, et al. A prospective study of work related factors and physical exercise as predictors of shoulder pain. Occup Environ Med 2001;58:528-34.

35 Andersen JH, Kaergaard A, Mikkelsen S, et al. Risk factors in the onset of neck/shoulder pain in a prospective study of workers in industrial and service companies. Occup Environ Med 2003;60:649-54.

36 Harkness EF, Macfarlane GJ, Nahit ES, et al. Mechanical and psychosocial factors predict new onset shoulder pain: a prospective cohort study of newly employed workers. Occup Environ Med 2003;60:850-7.

37 Leclerc A, Landre M-F, Chastang JF, et al. Upper-limb disorders in repetitive work. Scand J Work Environ Health 2001 ;27:268-78.

38 Leclerc A, Chastang JF, Niedhammer I, et al. Incidence of shoulder pain in repetitive work. Occup Environ Med 2004;61:39-44.

Answers to multiple choice questions on Current concepts of irritant contact dermatitis by J S C English, on pages 722-726

(1) $\mathrm{C}$

(2) $A$

(3) D

(4) D

(5) D 